The Astrophysical Journal, 548:L87-L89, 2001 February 10

(C) 2001. The American Astronomical Society. All rights reserved. Printed in U.S.A.

\title{
DISCOVERY OF VARIATION IN SOLAR CORONAL ROTATION WITH ALTITUDE
}

\author{
Hari Om Vats, ${ }^{1,2}$ J. R. Cecatto,,${ }^{1}$ M. Mehta, ${ }^{3}$ H. S. Sawant,${ }^{1}$ and J. A. C. F. Neri ${ }^{1}$ \\ Received 2000 August 30; accepted 2000 November 2; published 2001 February 2
}

\begin{abstract}
Here we report the first measure in radio emission of differential rotation as a function of height in the solar corona. This is derived from the disk-integrated simultaneous daily measurements of solar flux at 11 radio frequencies in the range of $275-2800 \mathrm{MHz}$. Based on the model calculations, these radio emissions originate from the solar corona in the estimated average height range of $\sim(6-15) \times 10^{4} \mathrm{~km}$ above the photosphere. The investigations indicate that the sidereal rotation period at the highest frequency $(2800 \mathrm{MHz})$, which originates from the lower corona around $6 \times 10^{4} \mathrm{~km}$, is $\sim 24.1$ days. The sidereal rotation period decreases with height to $\sim 23.7$ days at the lower frequency $(405 \mathrm{MHz})$, which originates at $\sim 13 \times 10^{4} \mathrm{~km}$. It is difficult to identify clearly the rotational modulation at $275 \mathrm{MHz}$, perhaps because these emissions are significantly affected by the turbulence in the intervening medium. Since these investigations are based on disk-integrated solar flux at radio frequencies, it is difficult to say whether these systematic variations in sidereal rotation period are partly due to the latitudinal differential rotation of the solar corona. It will be interesting to investigate this possibility in the future.
\end{abstract}

Subject headings: Sun: corona — Sun: radio radiation — Sun: rotation

\section{INTRODUCTION}

Solar rotation was discovered about four centuries ago by Galileo, but for more than two centuries the problem of solar rotation was practically ignored. Around 1850, Richard Carrington and Gustav Sporer independently confirmed that the visible envelope of the Sun does not rotate like a solid body; i.e., its period of rotation varies as a function of heliocentric latitude, with rotation being at a minimum at the equator and increasing gradually toward the poles. The measurements of solar rotation have been carried out mainly by using two methods, namely, (1) by tracing the passage of semipermanent features across the solar disk, e.g., sunspots, faculae, filaments, etc., and (2) by the spectroscopic observations of Doppler displacements of selected spectral lines. Each of these methods has its own limitations. The observations and their interpretations are excellently reviewed by Howard (1996 and references therein). Large volumes of data exist today, but the phenomenon of solar rotation is far from understood.

The rotation of the solar corona is even less understood, mainly because (1) its features are less distinct in duration and extent, (2) the corona is optically thin across a wide range of observed frequencies, and (3) it is generally difficult to measure directly the magnetic field there. Coronal rotation has been measured using emissions in white light, the Fe XIV green line, soft X-rays, and radio. Hansen, Hansen, \& Loomis (1969), using the K-coronameter, found a nearly constant rotation rate for heights ranging from 1.125 to 2 solar radii in 1967. Parker, Hansen, \& Hansen (1982), using similar observations during solar cycle 20 , observed a general increase in the coronal rotation rate. Sime, Fisher, \& Altrock (1989) used synoptic photoelectric observations of the coronal Fe XIV line at $5303 \AA$ and reported that their rotation properties are more similar to the white-light corona than to those of active regions on the Sun with which they are generally thought to be associated. The analysis of the Large-Angle Spectrometric Coronagraph

\footnotetext{
${ }^{1}$ Divisão de Astrofísica, Instituto Nacional de Pesquisas Espaciais, Avenida dos Astronautas, 1758, São José, dos Campos, Sao Paulo, 12201-970, Brazil; vats@das.inpe.br.

${ }^{2}$ On leave from the Physical Research Laboratory, Navrangpura, Ahmedabahd, 380-009, India.

${ }^{3} \mathrm{~J}$ and $\mathrm{J}$ Science College, Nadiad, Gujarat, India.
}

CI images shows that the global emission pattern rotates quasirigidly at the rate of the dominant active region complex (Wang et al. 1997). Inhester et al. (1999) also find no significant deviation from a rigidly rotating Fe XIV line with latitude or with distance from the Sun.

Skylab soft X-ray observations of an elongated coronal hole, which extended from the north pole to a latitude of $\sim 20^{\circ}$ south, show the almost rigid rotational characteristics of this feature (Timothy, Krieger, \& Vaiana 1975). Kozuka et al. (1994) investigated large-scale coronal structures using Yohkoh soft Xray observations and found that the rotation periods in the northern hemisphere are different from those in the southern hemisphere. Similarly, the corona is found to have a small but measurable latitudinal gradient in the rotation rate (Weber et al. 1999).

Aurass, Kurths, \& Voigt (1978) found a rigid rotation of emitting regions for the 1 and $9.4 \mathrm{GHz}$ radio flux. Vats et al. (1998a) did a fractal analysis of the solar radio emissions in the frequency range from $245 \mathrm{MHz}$ to $15 \mathrm{GHz}$ and found that the fractal dimension is least at $\sim 3 \mathrm{GHz}$ and increases significantly toward higher and lower frequencies. This implies that the radio emissions at $\sim 3 \mathrm{GHz}$ are least affected by the irregularities and random activities within the solar atmosphere as well as in the intervening medium. Based on this, Vats et al. (1998b) demonstrated that the time series of radio emission at $2.8 \mathrm{GHz}$ has a very strong modulation due to the solar coronal rotation, and thus they used the data of solar cycle 22 to determine the coronal rotation period. There exist almost continuous daily measurements of solar radio flux at 275, 405, 670, $810,925,1080,1215,1350,1620$, and $1755 \mathrm{MHz}$ (from the Cracow Astronomical Observatory) ${ }^{4}$ and at $2800 \mathrm{MHz}$ (from the Algonquin Radio Observatory in Canada). ${ }^{5}$ These data sets for a period of 26 months (1997 June 1-1999 July 31) are used here to estimate the coronal rotation in this height range. The analysis and results are presented in the next section.

\footnotetext{
${ }^{4}$ See http://www.oa.uj.edu.pl/sol/index.html.

${ }^{5}$ Available through the National Geophysical Data Center of the National Oceanic and Atmospheric Administration (http://www.ngdc.noaa.gov).
} 